\title{
EFFECTS OF SEGREGATION IN NICKEL-BASE SUPERALLOYS: DENDRITIC STRESSES
}

\author{
Alexander Epishin ${ }^{1}$, Thomas Link ${ }^{2}$, Udo Brückner ${ }^{1}$, Bernard Fedelich ${ }^{1}$ and Pedro Portella ${ }^{1}$ \\ ${ }^{1}$ Federal Institute for Materials Research and Testing, \\ Unter den Eichen 87, 12205 Berlin, Germany \\ ${ }^{2}$ Technical University Berlin, Institute for Material Science and Engineering, BH 18, \\ Ernst Reuter Platz 1, 10587 Berlin, Germany
}

Keywords: Nickel-base superalloys, Dendritic structure, Segregation, Residual stresses

\begin{abstract}
Residual stresses in the dendritic structure of single-crystal nickelbase superalloys were investigated. Dilatometric measurements showed that the thermal contraction of the dendrite arms (DAs) is larger than that of the interdendritic regions (IRs). The reason is, that the $\gamma^{\prime}$ phase, precipitating during cooling, has a smaller lattice parameter than the $\gamma$-matrix and that this $\gamma / \gamma^{\prime}$ misfit is higher in the DA than in the IR. In the "DA-IR-compound" the different thermal contractions cause residual stresses. The stress distribution within the dendritic cell was analyzed theoretically by finite element (FE) modeling. The stresses were proved directly by X-ray diffraction (XRD) and indirectly by the transformation of the $\gamma / \gamma^{\prime}$ morphology observed after load free annealing. It was found that dendritic stresses influence the structural and mechanical behavior of superalloys during high temperature creep: rafting and plastic deformation start in the primary dendrite arms (PDA).
\end{abstract}

\section{Introduction}

Dendritic segregation in nickel-base superalloys is a side effect of solidification. It is especially pronounced in superalloys of the newest generations with high levels of slowly diffusing refractory elements. Because the segregation of these elements (especially rhenium) can not be fully removed within acceptable homogenization time, superalloys are used with significant residual segregation. Therefore, it is important to know, how segregation affects the mechanical and structural behavior of superalloys. Two effects, inhomogeneity of the microstructure and phase instability of superalloys (precipitation of topologically closed packed phases) are widely investigated. This paper presents another segregation effect, namely residual stresses within the dendritic cell, "dendritic stresses", which are thermal stresses arising during cooling of superalloys due to the different thermal contraction of DAs and IRs.

\section{Materials and Methods}

The dendritic stresses were investigated in [001] single-crystals of CMSX-4 solidified and heat treated by Doncasters Precision Castings (DPC), Bochum, Germany. Analysis of the macrostructure of CMSX-4 shows, that after dissolving of the $\gamma / \gamma$ ' eutectics a significant residual segregation of the slowly diffusing elements rhenium and tungsten still remains. Figure 1 shows the inhomogeneous distribution of rhenium in heat treated CMSX-4. The rhenium concentration in the PDA is almost two times higher than in the IR. It was shown in [1] that segregation of rhenium and tungsten in CMSX-4 results in an inhomogeneity of the $\gamma / \gamma$ ' misfit: PDA: $-0.19 \%$, SDA: $-0.12 \%$ and IR: $+0.02 \%$ (unconstrained misfit). Accordingly two effects are expected: inhomogeneity of the coherency stresses and inhomogeneity of the thermal contraction during $\gamma$ '-precipitation. The second effect is the main interest of this investigation. It was checked experimentally by dilatometric analysis. Experimental data about the inhomogeneity of the misfit and the rhenium distribution allowed the modelling of the distribution of the resulting residual stresses within the dendritic cell. The theoretical results were validated by XRD. The effect of dendritic stresses on raft formation was investigated using scanning electron microscopy (SEM), their effect on dislocation mobility by transmission electron microscopy (TEM).

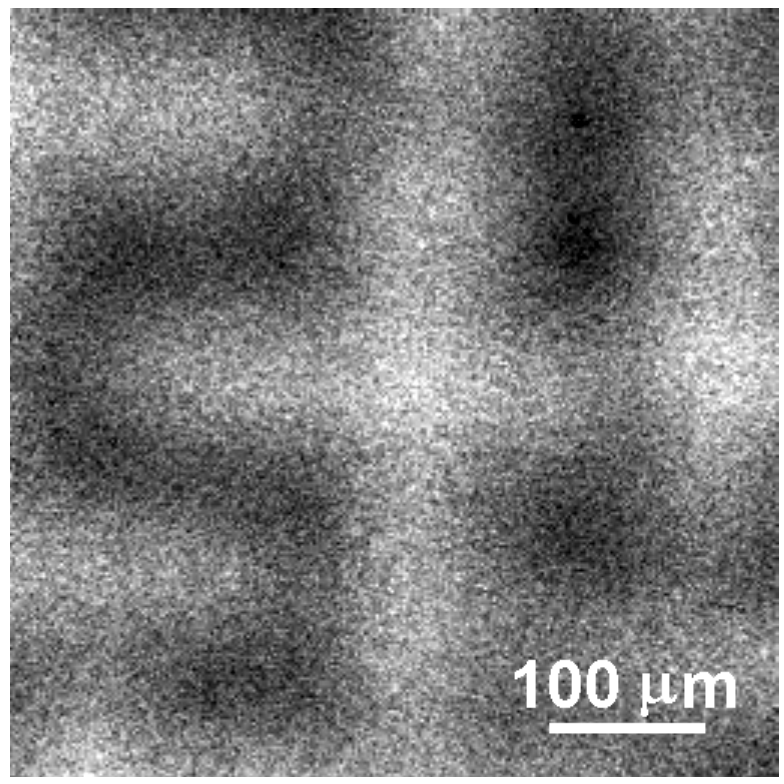

Figure 1. Segregation of Rhenium in the dendritic structure of fully heat treated CMSX-4. Rhenium concentration in PDA is about $4.5 \mathrm{wt} \%$, in IR about $2.4 \mathrm{wt} \%$.

\section{Results}

Investigation of Thermal Contraction

The influence of the misfit inhomogeneity on the thermal contraction was checked experimentally by casting two 
specimens, one with the composition of the PDA of heat treated CMSX-4, the other with that of the IR. The dilatometric analysis of these specimens showed that during cooling below the $\gamma$ ' solvus in the temperature range of $\gamma^{\prime}$ precipitation, the PDA contracts more than the IR (see Figure 2). After the $\gamma$ ' precipitation is finished, the dilatometric curves of the PDA and the IR specimens are equidistant. The total difference in the thermal contraction of these specimens during cooling from $\gamma^{\prime}$ solvus to room temperature was found to be about $\Delta \varepsilon^{T} \approx 0.1 \%$. This effect is directly related with the dendritic inhomogeneity of the $\gamma / \gamma^{\prime}$ misfit $\delta$. During cooling, the $\gamma^{\prime}$ precipitation causes a contraction of the superalloy because the $\gamma^{\prime}$ phase has a smaller lattice spacing than the $\gamma$ matrix. The contraction is larger in the PDA where $|\delta|$ is higher than in the IRs. The difference in thermal contraction found between the PDA and the IR specimens fits well with the difference in misfit $\Delta \delta \approx-0.15 \%$ measured in these specimens by X-ray diffraction: estimating $\Delta \varepsilon^{T} \approx \Delta \delta \cdot V^{\prime}$, with $\gamma^{\prime}$ volume fraction $V^{\prime} \approx 0.7$ gives $\Delta \varepsilon^{T} \approx 0.1 \%$. Thus the dendritic inhomogeneity of the $\gamma / \gamma^{\prime}$ misfit results in different thermal contractions within the dendritic cell, causing residual stresses.

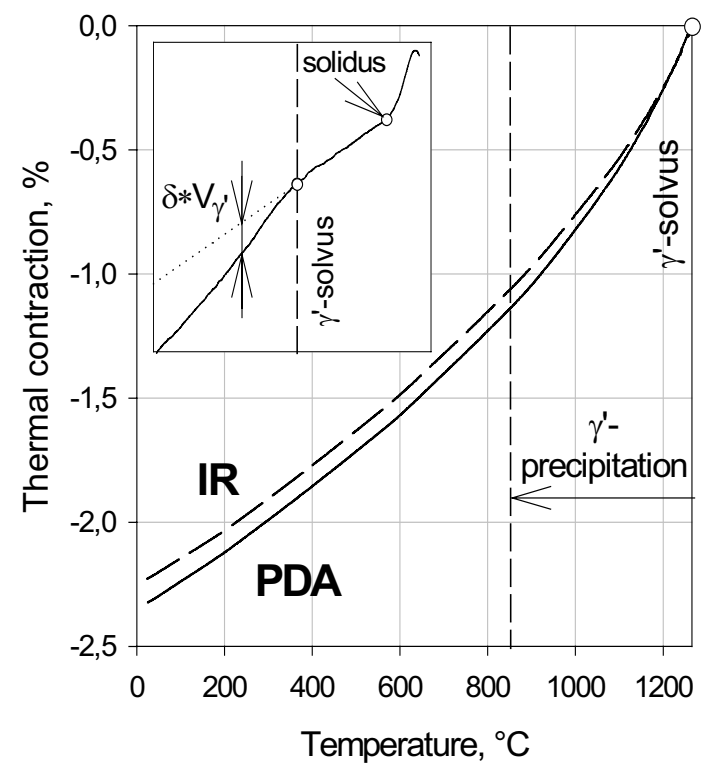

Figure 2. Thermal contraction of PDA and IR specimens during cooling below the $\gamma^{\prime}$ solvus. Insert: thermal contraction of CMSX-4.

\section{Modelling of Dendritic Stresses}

Modelling of dendritic stresses was performed using a geometrical model of the dendritic structure shown in Figure 3. The idealized single dendrite shows in a cross section a fourfold symmetry and in the longitudinal section an equidistant arrangement of the secondary dendrite arms. In [001] single-crystals such a dendrite is taken as a [001] columnar subgrain with $\{110\}$ boundaries. The subgrains are arranged in a quadratic lattice. From the symmetry it follows, that the periodic cell of the dendritic structure is a prism based on a equilateral right triangle with $<110>$ orientated short sides with the length $l=1 / 2 \sqrt{\rho^{P D A}}$, where $\rho^{P D A}$ is the area density of dendrites in a (001) section. The prism has the height (thickness) $t$ being equal to half the secondary dendrite arm (SDA) spacing $\lambda^{S D A} / 2$. Because the scale does not influence the results of the stress analysis, just the ratio $l / t=\left(\lambda^{S D A} \sqrt{\rho^{P D A}}\right)^{-1}$ is important. The quantitative metallographic investigation of the dendritic structure of CMSX-4 gave $\lambda^{S D A}=0.126 \mathrm{~mm}$ and $\rho^{P D A}$ $=12.5 \mathrm{~mm}^{-2}$, i.e. $l / t=2.25$. The calculations were performed using the FE code Abaqus [2], with boundary conditions according to the structure symmetry. The inhomogeneity of the thermal contraction $\varepsilon^{T}(\vec{x})$ was introduced as $\varepsilon^{T}(\vec{x})=\delta(\vec{x}) \cdot V^{\prime}$, where $\delta(\vec{x})$ describes the misfit distribution within the dendrite cell. The function $\delta(\vec{x})$ was designed on the basis of results of the misfit [1] and element [3] distribution in CMSX-4. It was assumed that above the $\gamma$ ' solvus all stresses in the dendritic structure relax during homogenization, i.e. the stress free state at the $\gamma^{\prime}$ solvus was taken as reference for FE modeling.

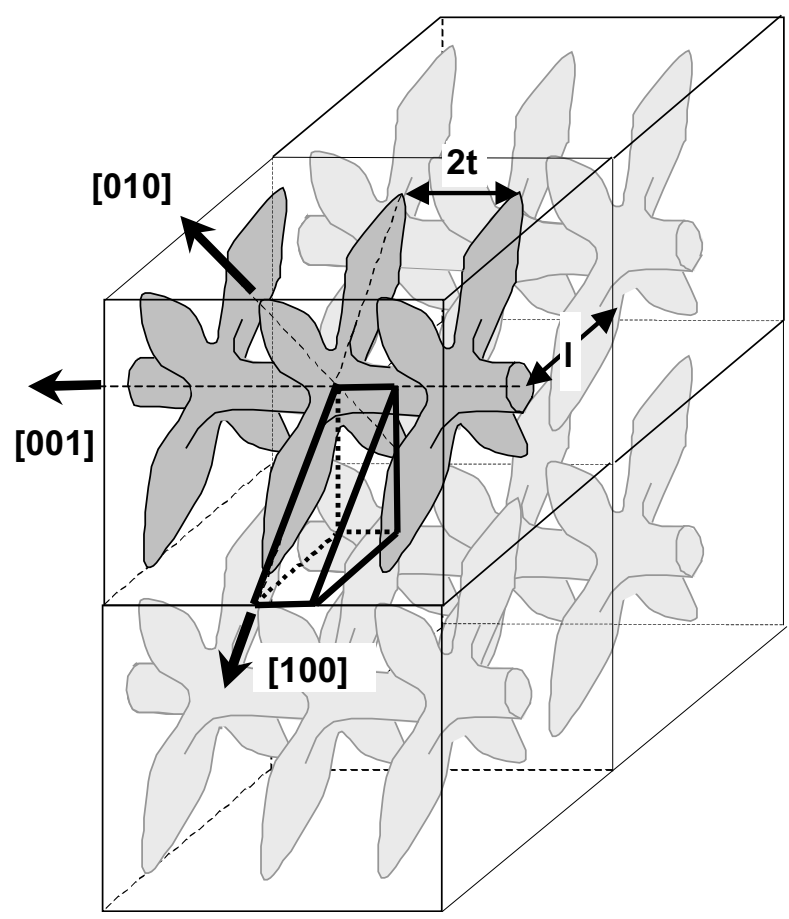

Figure 3. Computational domain schematically shown with respect to the dendritic structure.

Figures 4a-c show the distribution of the dendritic stresses at $20^{\circ} \mathrm{C}$. It is seen, that the components of the stress tensor $\sigma_{11}$, $\sigma_{22}$ and $\sigma_{33}$ are positive in the dendrite arms (the indices 1, 2, 3 correspond to the $<100>$ axes). In the PDA the largest component is $\sigma_{33} \approx+240 \mathrm{MPa}$ (Figure $4 \mathrm{c}$ ), in the SDA it is $\sigma_{11} \approx+140 \mathrm{MPa}$ (Figure 4a). It means that the DAs are in three dimensional (3D) tension with the strongest tensile component along the arm axis. 


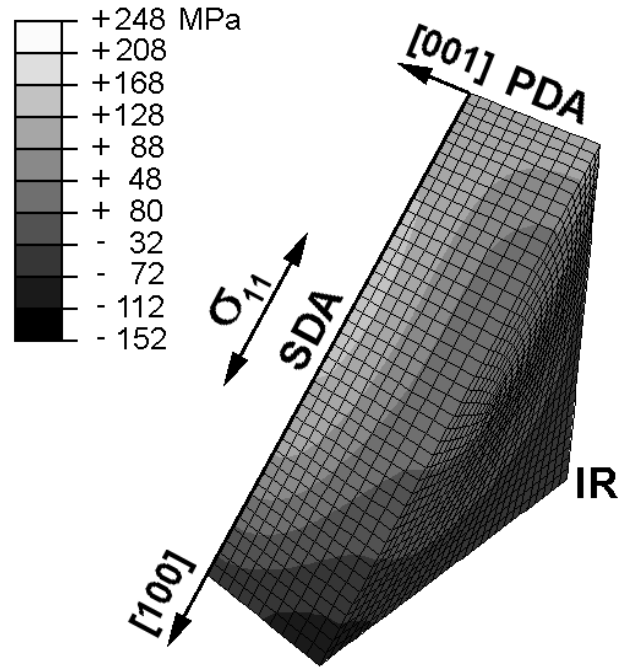

a

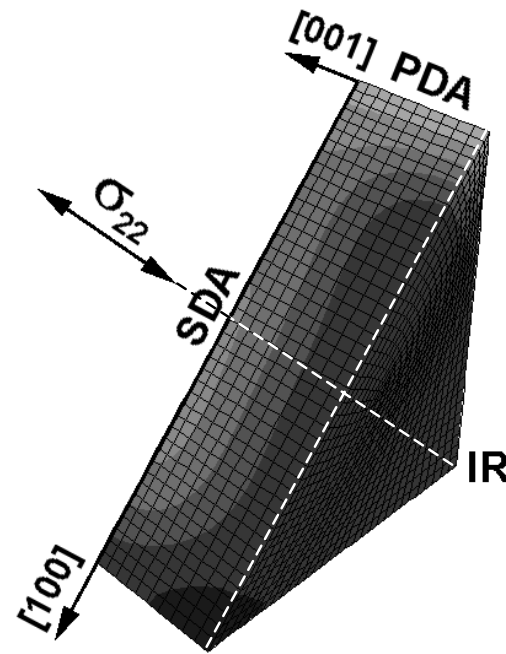

b

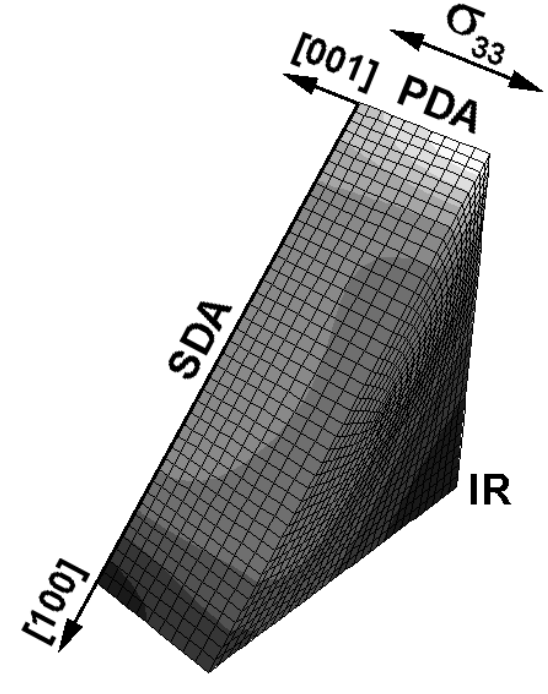

c

Figure 4. Distribution of the components of the dendritic stresses $\sigma_{11}, \sigma_{22}$ and $\sigma_{33}$ at $20^{\circ} \mathrm{C}$.

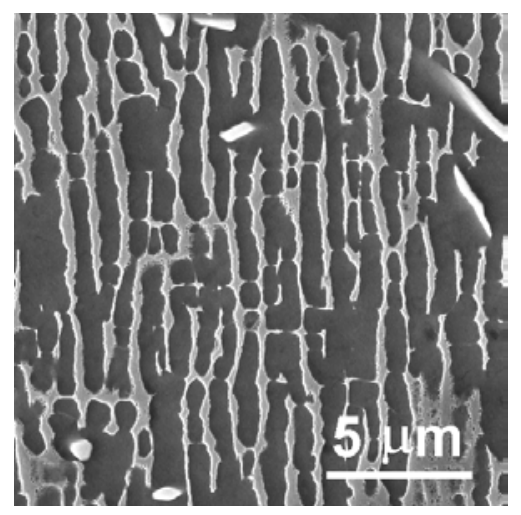

SDA

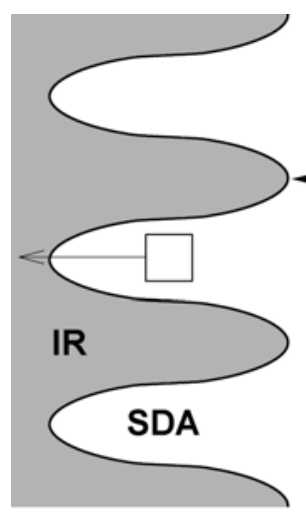

IR

吕

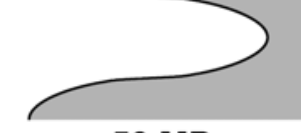

$50 \mathrm{MPa}$

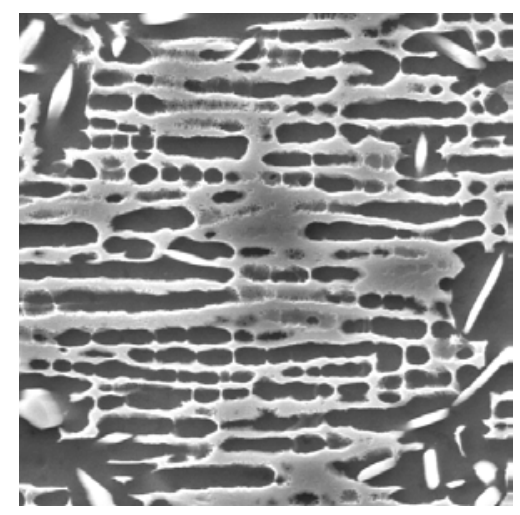

PDA

Figure 5. $\gamma / \gamma^{\prime}$ microstructure of CMSX-4 rafted during $300 \mathrm{~h}$ annealing at $1100^{\circ} \mathrm{C}$ under denritic stresses.

Double side arrows: dendritic stresses estimated by FE modeling.

Squares: positions of the SEM frames within the dendrite.

In the IR the stresses are negative, so the IR is under $3 \mathrm{D}$ compression. Calculation of the dendritic stresses at $850^{\circ} \mathrm{C}$ and $1100^{\circ} \mathrm{C}$ gave similar distributions but smaller absolute values of the stresses. At $850^{\circ} \mathrm{C}$ the stress values are about $80 \%$ of those at $20^{\circ} \mathrm{C}$, while at $1100^{\circ} \mathrm{C}$ they are about $45 \%$. This decrease of the stress values is mostly caused by the decrease of the elastic stiffness. At $1100^{\circ} \mathrm{C}$ the reduction of the $\gamma^{\prime}$ volume fraction makes an additional contribution in the decrease of the stress level.

\section{$\underline{\text { Rafting during Load Free Annealing }}$}

The occurrence of dendritic stresses has been proved indirectly by the transformation of the $\gamma / \gamma^{\prime}$ morphology observed after load free annealing. From rafting, it is known $[4,5]$ that rafts form perpendicular to the stress axis under tension and parallel under compression (for negative misfit). If no external load is acting, the residual stresses determine the raft formation, i.e. the raft morphology gives qualitative information about direction and spatial distribution of residual stresses. To reveal the dendritic stresses, specimens of fully heat treated CMSX-10, CMSX-4, SRR99 and SC16 were additionally annealed at $1100^{\circ} \mathrm{C}$ for $300 \mathrm{~h}$. After annealing the $\gamma / \gamma^{\prime}$ microstructure of each specimen was analyzed in SEM. The most pronounced rafting was found in CMSX-4, less in SRR99. In CMSX-10 rafting was observed only in the PDA and in SC16 no rafting at all. This result fits with the inhomogeneity of the misfit in these superalloys $[1,6]$ : for CMSX-4, SRR99, CMSX-10 and SC16 the difference of the $\gamma / \gamma$ ' misfit between PDA and IR is about $-0.21,-0.09,-0.07$ and $0 \%$ respectively. In all rafted superalloys the raft orientation in the DAs is perpendicular to the arm axis, i.e. perpendicular to the direction of the strongest tensile component of the dendritic stress (see Figure 5). Rafting in superalloys after load free annealing was observed also in $[7,8]$. 


\section{Measurement of the Lattice Strain by X-Ray Diffraction}

The occurrence of dendritic stresses has also been proved directly by local X-ray diffraction with a spatial resolution of about 100 $\mu \mathrm{m}$. The measurements were performed on a longitudinal cut, which was slightly tilted from the (100) plane around the [010] axis. So the dendrite axis is cut under a small angle, which makes it possible to observe the center of a dendrite and to position the X-ray spot within the dendritic structure (see Figure 6a). The tetragonal strain of the lattice unit cell, i.e. the difference $\Delta \varepsilon=\varepsilon_{3^{-}}$ $\varepsilon_{1}$ between the elastic strains along the PDA axis [001] $\left(\varepsilon_{3}\right)$ and along the perpendicular axis [100] $\left(\varepsilon_{1}\right)$ was analyzed. This

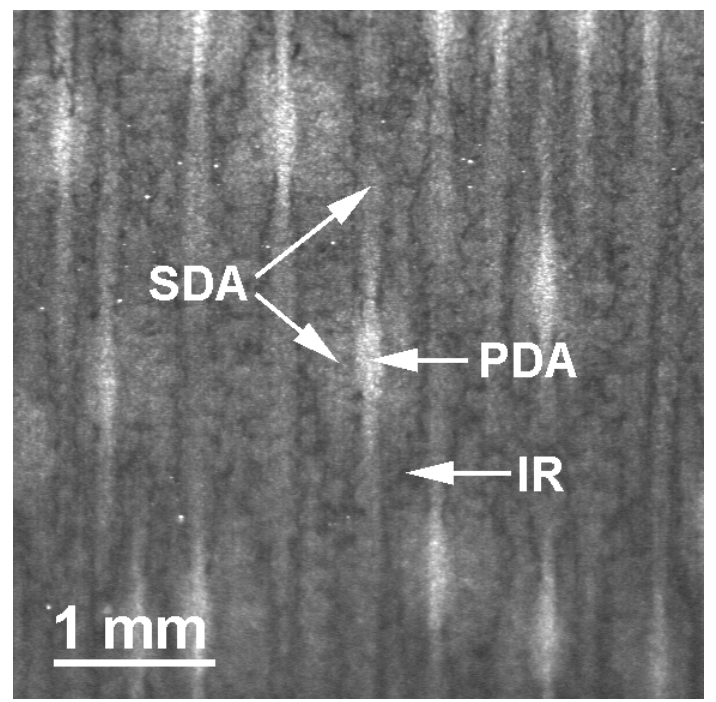

tetragonal strain can be determined by measuring the $\gamma^{\prime}$ lattice spacings $d_{h 0 l}$ of different $\{h 0 l\}$ planes belonging to the [010] zone axis. For small elastic strains it is valid:

$$
a_{\text {eff }}=a\left(1+\Delta \varepsilon \cdot \sin ^{2} \alpha\right)
$$

where $a_{e f f}=d_{h 0 l} \sqrt{h^{2}+l^{2}}$ is the effective lattice spacing, $a$ the lattice parameter in [100] direction and $\alpha$ the angle between [100] and $[h 0 l] . h$ and $l$ are Miller indices. Thus measuring $d_{h 0 l}$ as a function of $\sin ^{2} \alpha$ one can get $\Delta \varepsilon$. A detailed description of this method is presented in [9].

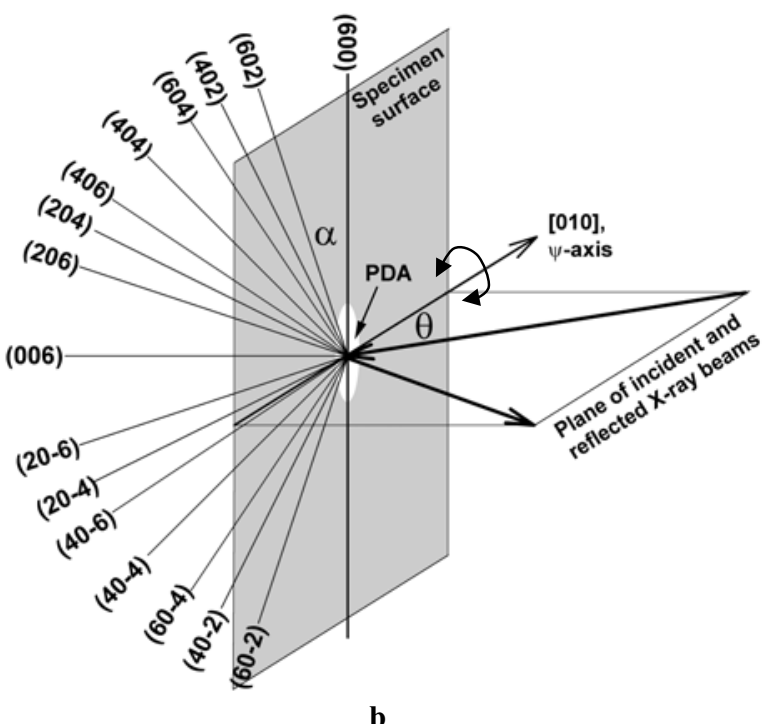

Figure 6. Analysis of the tetragonal strain in the PDA of CMSX-4 using a tilted longitudinal cut. a. - Image of the electropolished dendritic structure observed in X-ray diffractometer by CCD camera. b. - Geometry of the X-ray measurements: by rotation of the specimen around the [010] axis and adaptation of the Bragg angle the different lattice planes come into reflection position.
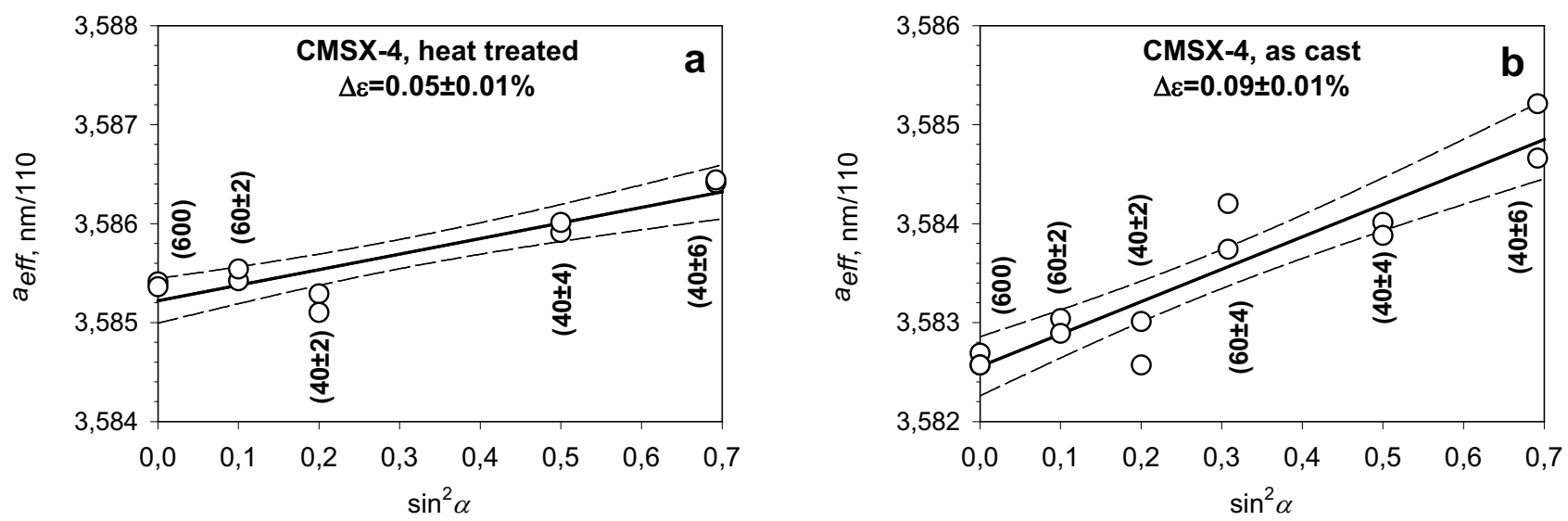

Figure 7. Effective lattice spacing $a_{\text {eff }}$ for plane orientations changing from parallel to the PDA axis $\left(\alpha=0^{\circ}\right)$ towards perpendicular $\left(\alpha=90^{\circ}\right)$, measured in the PDA. The dashed lines show the $95 \%$ confidential intervals. a. - Fully heat treated CMSX-4. b. - As cast CMSX-4. 
When measuring $\Delta \varepsilon$ it has to be taken into account that not only the dendritic stresses cause such a lattice distortion, but misfit stresses as well. In undeformed material the coherency stresses cause an isotropic tension in the $\gamma^{\prime}$ precipitates. Therefore for undeformed material only the $2 \theta$-position of the $\gamma^{\prime}$ peak of the $h 0 l$ $\mathrm{MoK} \alpha_{1}$ reflection was measured to determine $a_{\text {eff }}$. In crept material the $\gamma$ and $\gamma$ ' lattices have opposite tetragonal strains. For the average $\gamma / \gamma$ ' lattice spacing however this effect is compensated because the elastic constants of both phases are very close. Therefore for crept material the total $2 \theta$-profile of the $h 0 l \mathrm{MoK} \alpha_{1}$ reflection was measured and $a_{\text {eff }}$ was calculated by the $2 \theta$ position of the center of gravity of the profile.

The results for undeformed fully heated treated CMSX-4 are shown in Figure $7 \mathrm{a}$. The slope of the graph gives for $\Delta \varepsilon$ about $0.05 \%$, FE modelling $\Delta \varepsilon \approx 0.1 \%$. The reason for this difference is, that for X-ray measurements $\Delta \varepsilon$ is the average within the irradiated material (about $15 \times 80 \times 100 \mu^{3}$ ) whereas FE modelling gives the local maximum. Figure $7 \mathrm{~b}$ shows results for as cast CMSX-4, which give $\Delta \varepsilon$ of about $0.09 \%$, i.e. nearly two times larger than in heated treated condition. Thus the higher inhomogeneity results in stronger dendritic stresses. The analogous measurements where performed for a [001] singlecrystal of CMSX-4 crept at $1100^{\circ} \mathrm{C}$ and $120 \mathrm{MPa}$ for $150 \mathrm{~h}$ (the middle of steady creep). The measurements gave $\Delta \varepsilon$ about $0.03 \%$, which means that dendritic stresses partially relax during creep.

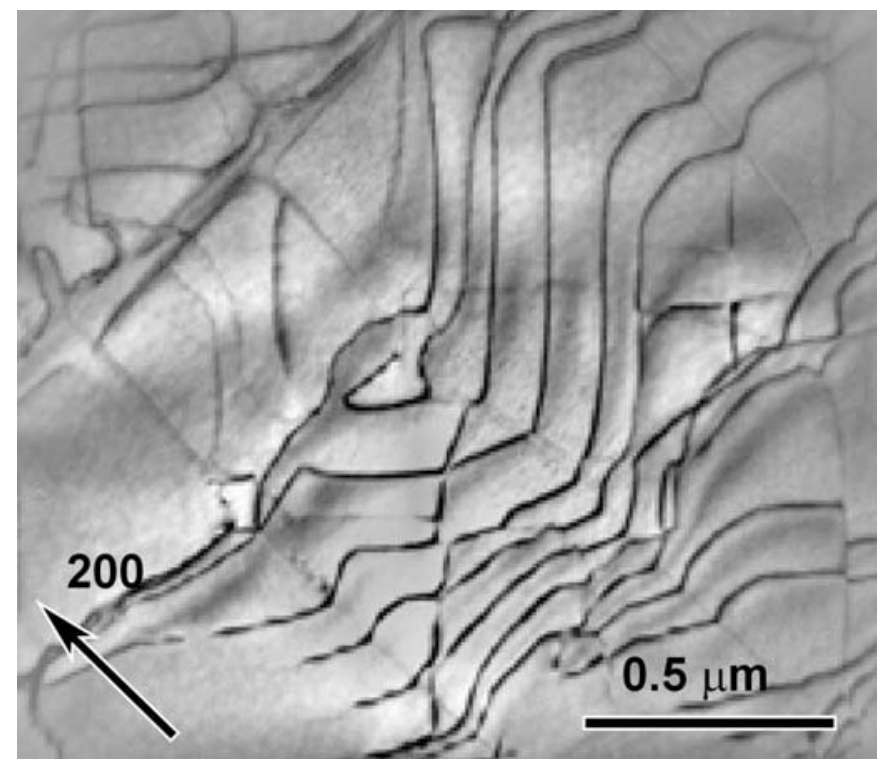

a

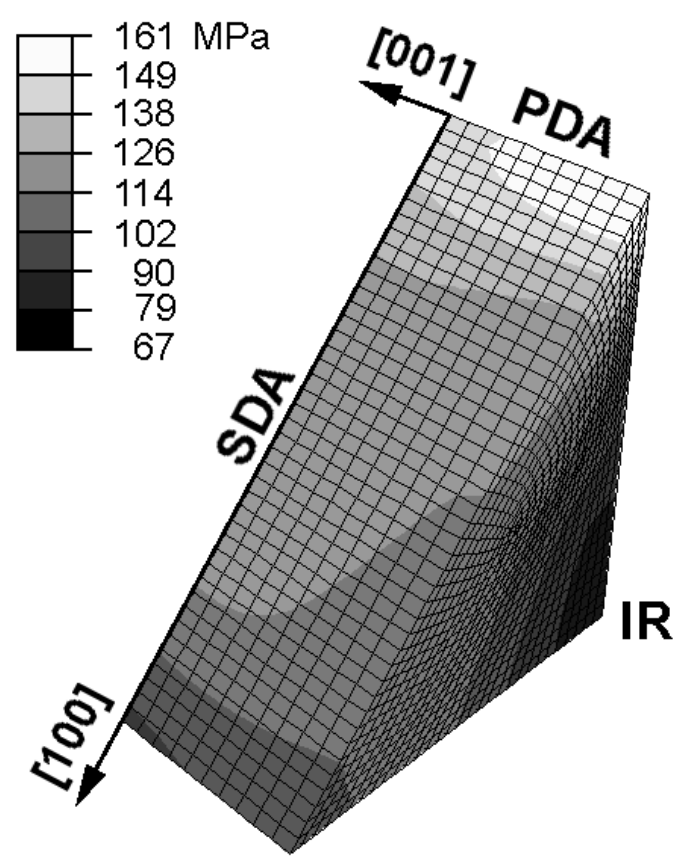

Figure 8. Distribution the RSS in the (001) oriented matrix channels in CMSX-4 at $1100^{\circ} \mathrm{C}$ immediately after loading by $120 \mathrm{MPa}$ stress in [001] direction.

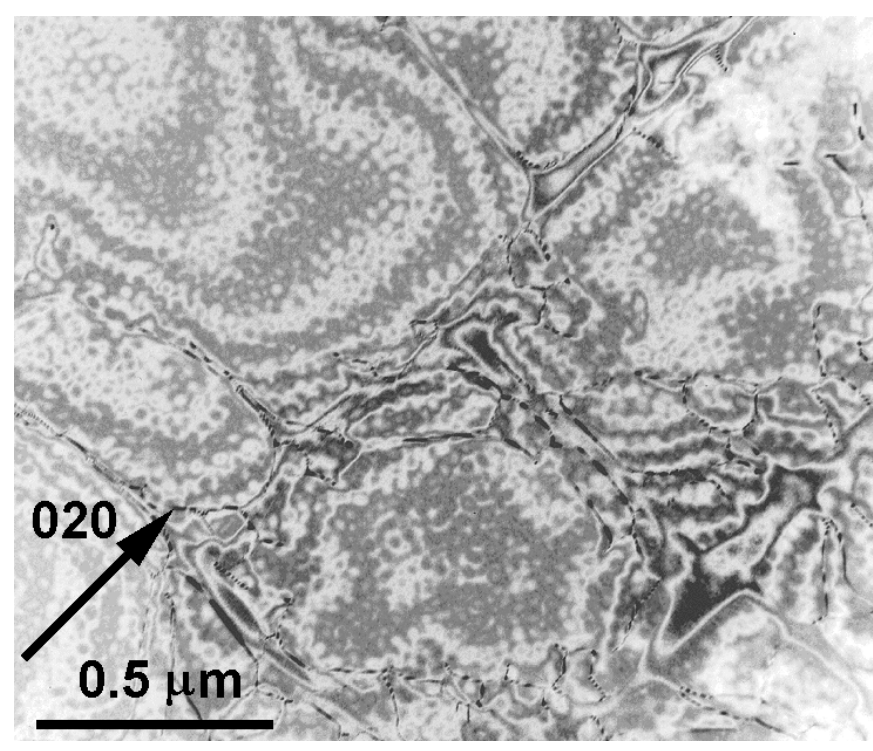

b

Figure 9. CMSX-4 after $4 \mathrm{~h}$ creep at $1100^{\circ} \mathrm{C}, 120 \mathrm{MPa}, \varepsilon=0.10 \%, \dot{\varepsilon}=3.8 \cdot 10^{-2} \% / \mathrm{h}$, TEM, (001) cross section. Dislocations in the $\gamma / \gamma^{\prime}$ interface indicating matrix glide in the PDA (a), in the IR (b) dislocations are rare.

\section{Inhomogeneity of Rafting and Plastic Deformation during Creep}

FE modelling of the stress distribution within the dendritic cell under uniaxial stress in [001] direction showed that it is strongly inhomogeneous. Figure 8 shows the distribution of the resolved shear stress (RSS) for the $<011>\{111\}$ glide system in the (001) matrix channels of CMSX-4 during creep at $1100^{\circ} \mathrm{C}$ and $120 \mathrm{MPa}$ immediately after loading in [001] direction. The model considered the two phase microstructure of the superalloy, i.e. the cuboidal $\gamma^{\prime}$ precipitates are coherently embedded in the $\gamma$ matrix. It is seen that the RSS in the PDA is about 2.5 times higher than in the IR. This effect is caused by two reasons: first, the 
coherency stresses are stronger in the PDA because $|\delta|$ is larger there, and second, in the PDA the applied tensile stress accumulates with the maximum tensile component of the dendritic stress. The result of this RSS concentration should be that plastic deformation starts in the matrix channels of the PDA. This supposition was checked by TEM investigations of

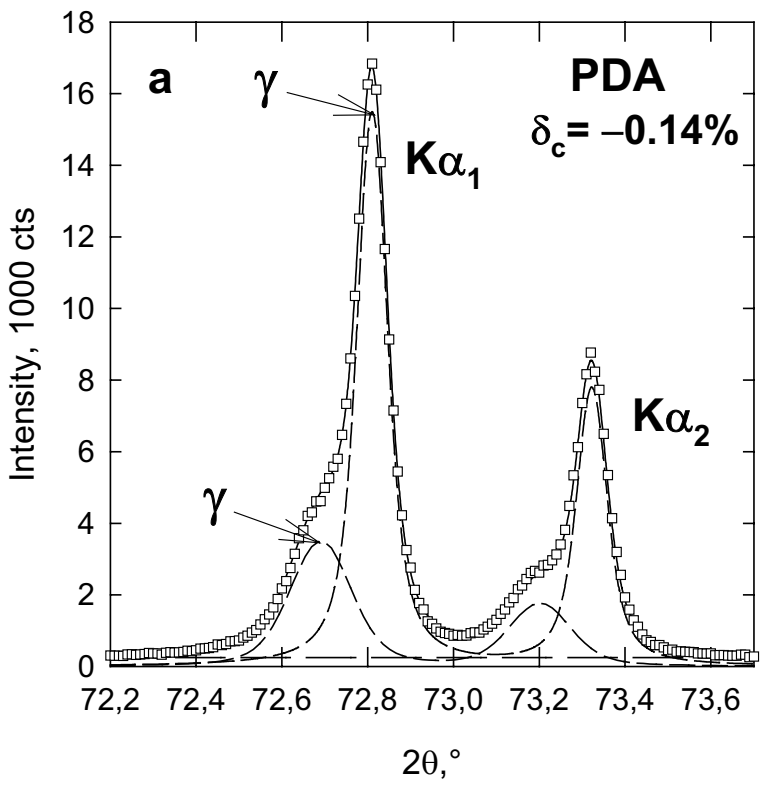

specimens at an early stage of deformation. Figure 9 shows the (001) interface in the PDA (a) and the IR (b) of CMSX-4 crept at $1100^{\circ} \mathrm{C}$ and $120 \mathrm{MPa}$ for $4 \mathrm{~h}$ (primary creep). In the PDA many interfacial dislocations deposited by matrix glide are found whereas in the IR they are quite rare, meaning that there is no remarkable matrix glide. This result was confirmed by X-ray

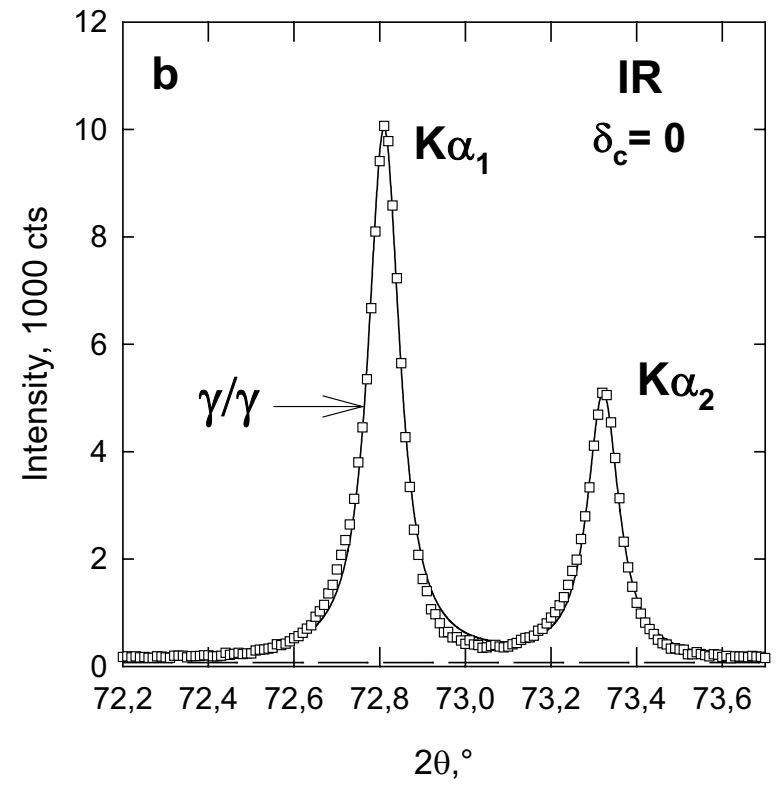

Figure 10: $2 \theta$ profiles of the 600 reflection of MoK $\alpha$ X-ray radiation measured in the PDA (a) and the IR (b) after $4 \mathrm{~h}$ creep at $1100^{\circ} \mathrm{C}$ and $120 \mathrm{MPa}$. Squares are experimental data; dashed lines are $\gamma$ and $\gamma$ ' subprofiles and background; solid lines are total fitted curves; $\delta_{\mathrm{c}}$ is the constrained $\gamma / \gamma^{\prime}$ misfit. Splitting of the $\gamma / \gamma^{\prime}$ peak in the PDA (a) indicates loss of coherency and no splitting in the IR (b) coherent interfaces.
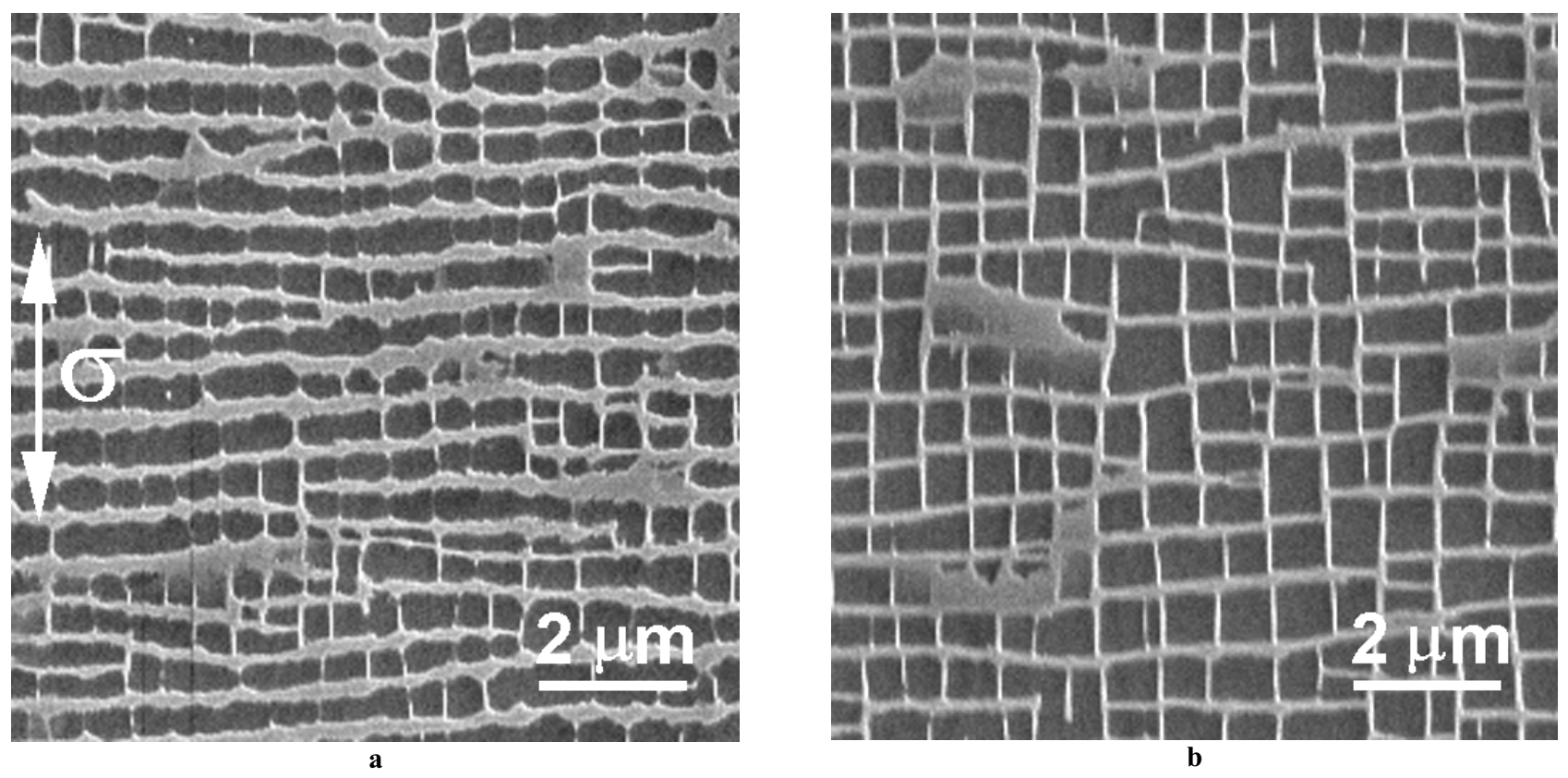

b

Figure 11. CMSX-4 after $4 \mathrm{~h}$ creep at $1100^{\circ} \mathrm{C}, 120 \mathrm{MPa}, \varepsilon=0.10 \%, \dot{\varepsilon}=3.8 \cdot 10^{-2} \% / \mathrm{h}$, SEM, longitudinal section (100). Rafting of the $\gamma / \gamma^{\prime}$-microstructure in the PDA (a), in the IR (b) the $\gamma / \gamma^{\prime}$ microstructure remains cuboidal. 
diffraction: clear splitting of the $\gamma / \gamma^{\prime}$ peak in the PDA (Figure 10a), which means loss of coherency and no splitting in the IR (Figure 10b) meaning, that the interfaces are still coherent. By SEM also structural changes, correlated to the stress inhomogeneity, were observed. After $4 \mathrm{~h}$ creep in the PDA rafting is nearly complete, while in the IR the $\gamma / \gamma^{\prime}$-microstructure is still cuboidal (compare Figures 11a and b). The results obtained for CMSX-4 are typical for such superalloys showing a significant residual segregation. The same differences in kinetics of dislocation glide, loss of coherency and rafting were found in the PDA and IR of CMSX-10 and SRR99 deformed during primary creep (CMSX-10: $1100^{\circ} \mathrm{C}, 120 \mathrm{MPa}, 25 \mathrm{~h}$ and $60 \mathrm{~h}$; SRR99: $980^{\circ} \mathrm{C}, 200 \mathrm{MPa}, 20 \mathrm{~h}$ )

It follows from the above results that segregation causes the misfit inhomogeneity and hereby it influences the primary creep of superalloys. This influence is quite complex (see Figure 12): the inhomogeneity of the coherency stresses and the dendritic stresses results in earlier plastic deformation in the PDA, which then expands over the entire dendritic cell. Exact statements about the influence of the segregation on the kinetics of creep process are difficult, but two effects should be expected: an initiation of the primary creep by earlier plastic deformation in the PDA and a non-monotonic change of the strain rate due to a step-like development of the plastic deformation within the dendritic cell $(\mathrm{PDA} \rightarrow \mathrm{SDA} \rightarrow \mathrm{IR})$. Such primary creep behavior was observed in $[3,10,11]$. Obviously FEM modelling is necessary for detailed understanding of the segregation effects.

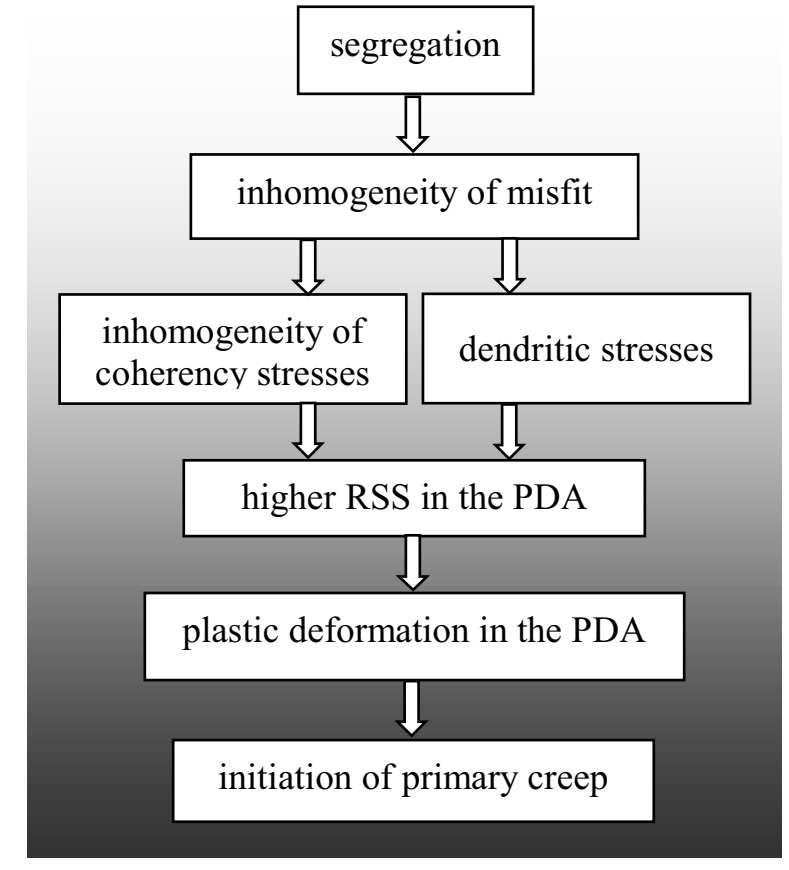

Figure 12. Influence of segregation on primary creep in superalloys

\section{Summary}

Residual stresses in the dendritic structure of superalloys were proved by various methods. They are caused by the different thermal contraction of DA and IR having different composition. The dendritic stresses result in an inhomogeneous 3D stress field which influences the mechanical and structural behaviour of superalloys. It is expected that this influence becomes relevant at low stress levels comparable with the dendritic stresses.

\section{Acknowledgements}

The authors are grateful to the Deutsche Forschungsgemeinschaft for financial support of this work (Project Po 405/4, Li 494/3).

\section{Rerefences}

1. U. Brückner et al., "Local X-ray diffraction analysis of the structure of dendrites in single-crystal nickel-base superalloys", Acta mater., 45 (1997), 5223-5231.

2. Abaqus, Version 6.3, (2002). Hibitt, Karlsson \& Sorensen, Inc. 1080 Main Street, Pawtucket, RI 02860-4847, http://www.abaqus.com

3. A. Epishin et al., "Influence of dendritic inhomogeneity on creep behavior of a single-crystal superalloy of the third generation" (Final report, Project Po 405/4, Li 494/3, Federal Institute of Materials Research and Testing - Technical University Berlin, 2004).

4. J. K. Tien and S. M. Copley, "The effect of uniaxial stress on the periodic morphology of coherent gamma prime precipitates in nickel-base superalloy crystals", Metall. Trans., 2 (1971), 215-219.

5. J. K. Tien and S. M. Copley, "The effect of orientation and sense of applied stress on the morphology of coherent gamma prime precipitates in stress annealed nickel-base superalloy crystals", Metall. Trans., 2 (1971), 543-553.

6. A. Epishin et al., "Influence of dendritic inhomogeneity on creep behavior of a single-crystal superalloy of the third generation" (Interim report, Project Po 405/4, Li 494/3, Federal Institute of Materials Research and Testing - Technical University Berlin, 2002).

7. A. Hazotte and J. Lacaze, "Chemically oriented $\gamma$ ' plate development in a nickel base superalloy", Scripta Metall., 23 (1989), 1877-1882.

8. A. Hazotte and A. Simon, "Quantitative analysis of the structural changes during aging of single crystal nickel-based superalloys", Acta Stereol., 8 (1989), 175-180.

9. U. Brückner et al., "Dendritic stresses in nickel-base superalloys" (Paper to be presented at the 7th Conference on Residual Stresses, Xian, China, 14-17 June 2004).

10. W. Schneider, „High temperature behavior and microstructure of single-crystal nickel-base superalloy CMSX-4 at temperatures from $800^{\circ} \mathrm{C}$ up to $1100^{\circ} \mathrm{C}$ ", $\mathrm{PhD}$ thesis, Universität Erlangen-Nürnberg, 1993.

11. F. Diologent et al., "Creep behavior at $1050^{\circ} \mathrm{C}$ of a new generation single crystal superalloy", Creep Deformation: Fundamentals and Applications, Ed. by R. S. Mishra, J. C. Earthman and S. V. Raj, TMS (The Minerals, Metals \& Materials Society), 2002, pp. 361-370. 
